Mens

revue d'histoire intellectuelle de l'Amérique française

\title{
D'une société de secours mutuel locale à une société fraternelle nationale : la transformation du projet social de l'Union Saint-Joseph d'Ottawa (1863-1905)
}

\section{Pierrick Labbé}

Volume 11, numéro 1, automne 2010

URI : https://id.erudit.org/iderudit/1023337ar

DOI : https://doi.org/10.7202/1023337ar

Aller au sommaire du numéro

Éditeur(s)

Centre de recherche en civilisation canadienne-française

ISSN

1492-8647 (imprimé)

1927-9299 (numérique)

Découvrir la revue

Citer cet article

Labbé, P. (2010). D'une société de secours mutuel locale à une société fraternelle nationale : la transformation du projet social de l'Union Saint-Joseph d'Ottawa (1863-1905). Mens, 11(1), 37-75.

https://doi.org/10.7202/1023337ar

\section{Résumé de l'article}

Cet article examine la transformation de la mutualité canadienne-française à la fin du xix ${ }^{\mathrm{e}}$ siècle en analysant le cas de l'Union Saint-Joseph d'Ottawa. Elle fut l'une des rares sociétés de secours mutuel de première génération à adapter sa structure et son projet social pour se conformer au courant mutualiste émergent et, ainsi, se transformer en une société fraternelle. L'exemple nous montre que la lutte pour la survivance dans les milieux de migration facilitait cette transition du modèle mutualiste et justifiait la redéfinition des objectifs par une petite élite au nom d'un idéal national. Le besoin d'assurer des bases institutionnelles solides pour les Canadiens français de l'Ontario légitimait cette transformation de l'Union Saint-Joseph, qui devint une grande société fraternelle vouée à la défense des intérêts économiques des Canadiens français, grâce à l'établissement de succursales dans plus de six cents communautés canadiennes-françaises du Canada et des États-Unis. 


\title{
Article
}

\section{D'une société de secours mutuel} locale à une société fraternelle nationale : la transformation du projet social de l'Union Saint-Joseph d'Ottawa (1863-1905)

\author{
Pierrick Labbé \\ Université d'Ottawa
}

\section{Résumé}

Cet article examine la transformation de la mutualité canadiennefrançaise à la fin du xix e siècle en analysant le cas de l'Union SaintJoseph d'Ottawa. Elle fut l'une des rares sociétés de secours mutuel de première génération à adapter sa structure et son projet social pour se conformer au courant mutualiste émergent et, ainsi, se transformer en une société fraternelle. L'exemple nous montre que la lutte pour la survivance dans les milieux de migration facilitait cette transition du modèle mutualiste et justifiait la redéfinition des objectifs par une petite élite au nom d'un idéal national. Le besoin d'assurer des bases institutionnelles solides pour les Canadiens français de l'Ontario légitimait cette transformation de l'Union Saint-Joseph, qui devint une grande société fraternelle vouée à la défense des intérêts économiques des Canadiens français, grâce à l'établissement de succursales dans plus de six cents communautés canadiennes-françaises du Canada et des États-Unis. 


\section{Abstract}

This article examines the transformation of French Canadian mutuality in the late nineteenth century through an examination of Ottawas Union Saint-Joseph. The Union was one of only a handful of first-generation mutual aid societies to adapt its structure and social project in order to conform to the emerging current of mutualism and transform itself into a fraternal society. Its example shows that the struggle for cultural survival in emigrant communities facilitated the transition of the mutualist model and justified the redefinition of its aims by a small elite in the name of a national ideal. The need to insure solid institutional bases for the French Canadians of Ontario legitimised the transformation of the Union Saint-Joseph, which became a large fraternal society devoted to defending the economic interests of French Canadians through the establishment of branches in over six hundred French Canadian communities in Canada and the United States.

À partir de la deuxième moitié du xix siècle, la mutualité sous la forme de sociétés de secours mutuel s'imposa comme le principal mode de prévoyance des classes populaires canadiennes-françaises. Plus d'une centaine de ces petites organisations furent fondées entre 1850 et 1900, dont plusieurs s'inspirèrent du modèle organisationnel de l'Union Saint-Joseph de Montréal'. Lors de la mise sur pied de cette société par des tailleurs de pierre en 1851, ses fondateurs

\footnotetext{
Le nombre exact de sociétés de secours mutuel locales fondées au cours de cette période est inconnu puisque l'enregistrement n'était pas obligatoire avant 1893 en Ontario et 1909 au Québec. Il s'agit d'une estimation tirée des recherches d'Yvan Rousseau, qu'il juge lui-même prudente. Martin Petitclerc, quant à lui, estime à environ 250 le nombre de sociétés de secours mutuel fondées au Québec au XIX ${ }^{e}$ siècle. Yvan Rousseau, « De la cotisation à la prime d'assurance : le secours mutuel au Québec entre 1880 et 1945 ", dans Alain Croix et Patrick Harismendy (dir.), Espaces et histoire : colloque franco-québécois, Université Rennes 2, Haute-Bretagne, 20-21 mai 2003, Rennes, Presses universitaires de Rennes, 2003, p. 151-152 ; Martin Petitclerc, "Nous protégeons l'infortune" : les origines populaires de l'économie sociale au Québec, Montréal, VLB éditeur, 2007, p. 12.
} 
codifièrent une structure administrative fondée sur des objectifs qui dépassaient les limites de l'activité économique. Le fonctionnement de l'organisation répondait à des idéaux d'entraide et d'égalité, formant un projet social qui visait à compenser la rupture des liens familiaux et ruraux entraînée par l'urbanisation. Ces valeurs influencèrent les premiers mutualistes lors de l'élaboration d'un système de gestion des finances. Le modèle prévoyait qu'en échange d'un paiement mensuel égal pour tous et d'une présence aux activités obligatoires, les membres de ces associations pourraient obtenir une protection financière pour leur famille en cas de décès ou d'accident. La participation aux assemblées, qui se déroulaient suivant des principes démocratiques, offrait la possibilité à tous les sociétaires d'intervenir pour influencer le processus décisionnel. Une constitution et des règlements codifiaient cette structure pour protéger ses valeurs d'égalité, permettant ainsi la conservation de l'idéologie fondatrice et la diffusion de l'idéal de solidarité populaire.

Ces petites organisations locales demeurèrent le mode de prévoyance de prédilection des classes moins nanties jusqu'à leur déclin à la fin du XIX ${ }^{\mathrm{e}}$ siècle. Leur disparition s'explique, en partie, par la fragilité de leur gestion financière, qui les rendait vulnérables aux aléas de l'économie. De plus, les Canadiens français se tournèrent graduellement vers les sociétés fraternelles à partir des années 1880 . Ces grandes associations, inspirées du modèle anglo-saxon des friendly societies, se distinguaient de la première génération de mutuelles par leur réseau de succursales, une administration centralisée et une gestion des finances plus rigoureuse. Leurs activités se multipliaient dans les centres urbains et ralliaient un nombre grandissant de sociétaires. Les sociétés fraternelles connaissaient une période de croissance ainsi qu'une stabilité supérieure aux anciennes sociétés de secours mutuel grâce à un recrutement intensif et à une répartition du risque parmi un plus grand nombre de cotisants. À la différence de la première génération de mutualistes, qui visait avant tout à secourir les classes populaires, leur mission sociale s’appuyait généralement 
sur un message national ou religieux ${ }^{2}$. De plus, à partir des années 1890 , plusieurs de ces associations adoptèrent des méthodes scientifiques et individuelles de calcul du risque ${ }^{3}$. Cette transition de l'économie mutualiste vers les sociétés fraternelles s'opéra au détriment des idéaux d'entraide, de solidarité, de participation et d'égalité fixés par la classe populaire qui animaient les petites sociétés de secours mutuel locales. La nouvelle définition du projet social qui en découla dépassait la simple réforme administrative en redéfinissant entièrement les objectifs économiques et sociaux de la mutualité.

Comme l'affirme l'historien Martin Petitclerc, le changement de conception de l'idéal mutualiste ne s'opéra jamais pour beaucoup de membres des petites sociétés de secours mutuel locales, qui demeurèrent réfractaires aux mutations qui mettaient en péril les valeurs organisationnelles initiales. Leur réticence s'explique par la destruction du concept de "famille fictive " qu'aurait entraînée l'adoption de méthodes de gestion scientifique et individuelle du risque. La solidarité, l'égalité, l'entraide et le désir de perpétuer cette expérience freinaient les tentatives de réformes visant à implanter un calcul des cotisations personnalisées basé sur le risque individuel. Le modèle

2 L'Ancient Order of United Workmen, les Forestiers catholiques, Ia Catholic Mutual Benefit Association, le Canadian Order of Foresters et d'autres associations implantèrent leurs succursales à Ottawa entre les années 1880 et 1890, créant un climat de compétition avec les mutuelles, qui dépendaient du recrutement dans cette ville pour survivre. Jacques Grimard et Gaétan Vallières, Travailleurs et gens d'affaires canadiens-français en Ontario, Saint-Laurent, Études vivantes, 1986, p. 190 ; Catholic Mutual Benefit Association of Canada, C.M.B.A. Herald, 22 juillet 1891, Kingston, p. 8 ; The Ottawa Directory, vol. 11, 1884-1885, Ottawa, A. S. Woodburn, p. 304 ; The Ottawa Directory : 1891-1892, Ottawa, Might's Directory, p. 36.

3 Les sociétés de secours mutuel de première génération ne conservaient pas de réserve d'argent considérable, et les paiements qui s'effectuaient suivant les décès ne permettaient pas d'accumulation substantielle. La cotisation mensuelle pour les frais médicaux suffisait à peine à défrayer les indemnités remises aux malades. Cependant, la somme remise à la veuve était fixe et déterminée par les règlements, tout comme les paiements mensuels et post mortem. La variation du nombre de membres déterminait la capacité de l'organisation à stabiliser ses finances. 
des sociétés fraternelles représentait une différente forme de mutualité. Le grand nombre de sociétaires de ces associations diminuait le poids de l'individu dans le processus décisionnel et reléguait le pouvoir aux mains des dirigeants élus lors de congrès annuels. En conséquence, lorsque les sociétés de secours mutuel de première génération furent acculées à la faillite à cause de l'instabilité de leur méthode de gestion des finances et de la diminution de leur capacité de recrutement par rapport aux sociétés fraternelles, les membres choisirent, dans la plupart des cas, de conserver les anciennes structures organisationnelles. L'adoption de méthodes administratives plus commerciales signifiait, pour les premiers mutualistes, l'abandon du projet social pour lequel ils avaient œuvré. L'échec du modèle mutualiste initial et l'incapacité des petites sociétés de secours mutuel locales à s'adapter à la nouvelle réalité, à cause de leur désir de conserver le statu quo, poussèrent la plupart de ces associations vers la faillite à mesure qu'elles devaient faire face à leur insolvabilité. Ce fut le cas, d'ailleurs, de l'Union Saint-Joseph de Montréal, qui cessa ses activités en $1909^{4}$.

L'Union Saint-Joseph d'Ottawa fut l'une des rares sociétés de secours mutuel locales de première génération à adopter des réformes pour se tourner vers cette forme de mutualité. Après plus de trentedeux ans d'activité, ses sociétaires acceptèrent de modifier sa constitution pour permettre le développement de succursales dans les communautés canadiennes-françaises du Canada et des États-Unis. Cette réforme signifiait également une diminution du pouvoir des membres au profit d'une administration professionnelle dirigée par une élite locale. Conséquemment, c'est l'ensemble de son projet social qui fut redéfini par les nouveaux dirigeants. Ceux-ci formulèrent une mission basée sur la promotion du catholicisme social et du nationalisme canadien-français. Ils en profitèrent également pour établir une gestion individuelle du risque et remplacer les cotisations uniformes et fixes des premières années.

\footnotetext{
4 Martin Petitclerc dénombra plusieurs associations qui connurent aussi le même sort. Petitclerc, "Nous protégeons l'infortune ", p. 157-165, 185-189.
} 
Cet article a pour but d'examiner les raisons qui ont favorisé la transition de l'Union Saint-Joseph d'Ottawa d'une petite association locale à une grande société fraternelle nationale. Sa survie jusqu’à nos jours a contribué à la conservation d'un certain nombre de documents clés qui rendent possible cette analyse. Les sources pour l'étude de la mutualité au XIX $X^{e}$ siècle sont plutôt rares étant donné l'existence éphémère de la plupart des sociétés de secours mutuel et le peu d'intérêt des gouvernements pour l'encadrement de ces associations à cette période. Les procès-verbaux et les rapports annuels de l'Union Saint-Joseph, conservés au Centre de recherche en civilisation canadienne-française de l'Université d'Ottawa, offrent ainsi une perspective originale de la mutualité. Les documents sont encore plus nombreux pour analyser ses activités après 1895 , surtout grâce à la parution d'un journal mensuel envoyé gratuitement à tous les sociétaires, portant le nom de Bulletin Officiel ou Le Prévoyant, après 1900 , et à la publication de rapports annuels. La totalité des sources émane des activités officielles de l'association et nous en donne une image incomplète. En l'absence de documents plus personnels concernant les réformateurs, l'examen se limite à la mutation de l'association en tant que groupe.

Malgré ces défauts, l'analyse montre clairement que la mutualité canadienne-française, dans ces nouveaux milieux d'implantation, avait tendance à s'insérer dans un cadre associatif paroissial où l'identité nationale représentait un élément clé qui unissait ses participants. La survivance en tant que groupe légitimait les réformes qui permirent à l'élite de transformer l'Union Saint-Joseph d'Ottawa. D'autres facteurs issus des expériences locales propres à chacune des associations ont aussi influé sur le développement des sociétés de secours mutuel, que ce soit l'adoption de nouvelles lois, les mutations sociales et communautaires ou encore la concurrence des nouvelles formes de mutualité. Ces facteurs n'empêchèrent pas une certaine constance évolutive, remarquée au sein de la plupart des sociétés de secours mutuel fondées dans le cadre des mouvements migratoires. John Bodnar affirmait d'ailleurs, à propos des relations entre l'immigration 
et la mutualité, que les différents groupes nationaux qui migrèrent en Amérique formèrent des associations en territoire d'accueil en reproduisant des comportements associatifs issus de leur pays d'origine. Parmi elles se trouvaient des sociétés de secours mutuel et des sociétés fraternelles. Bodnar mentionne également que le pouvoir au sein de ces associations passa graduellement des membres à quelques dirigeants, qui en profitèrent pour en reformuler les objectifs idéologiques. Les élites firent alors de la mutualité un outil de développement national en terre d'accueil, au nom des intérêts du groupe ${ }^{5}$. Cette tendance résume relativement bien les grandes lignes des transformations de l'Union Saint-Joseph d'Ottawa, de son implantation à Ottawa jusqu'à la prise de contrôle par une petite élite canadienne-française. Yves Roby confirme d'ailleurs ce rôle de la mutualité en tant qu'outil national pour lutter contre l'assimilation dans le cas des FrancoAméricains. Ce dernier montre cette étroite relation entre les mutualistes canadiens-français, le clergé et les autres institutions dans ces milieux d'enracinement ${ }^{6}$. La survivance et le maintien de leur culture donnaient des arguments de taille à l'élite pour convaincre les sociétaires d'adopter des réformes.

Même si la migration des Canadiens français à Ottawa se trouvait très près du foyer d'origine, les mêmes caractéristiques que celles relevées par Bodnar et Roby sont présentes dans le cas de l'Union Saint-Joseph d'Ottawa. La première partie du texte explique d'ailleurs comment sa fondation s'insérait dans une tentative de reproduire en milieu d'accueil une institution issue du territoire d'origine. Cependant, son développement fut marqué, dans les premières décennies, par le contexte d'évolution en milieu minoritaire et les rivalités ethniques. Ainsi, lorsque le modèle administratif initial se montra inadéquat pour surmonter les aléas de l'économie et la

\footnotetext{
5 John Bodnar, The Transplanted: A History of Immigrants in Urban America, Bloomington, Indiana University Press, 1985, p. 124-128.

${ }^{6}$ Yves Roby, Les Franco-Américains de la Nouvelle-Angleterre : rêves et réalités, Sillery, Septentrion, 2000, p. 90-91.
} 
concurrence des sociétés fraternelles, l'idée de développer une association nationale pour défendre les intérêts des Canadiens français était acceptable pour la majorité des sociétaires, même si la transition amenait également la refonte des valeurs mutualistes. La dernière partie de l'article examine ce nouveau projet, tel que défini par l'élite qui prit le contrôle de l'Union Saint-Joseph à la suite des réformes au tournant du $\mathrm{Xx}^{\mathrm{e}}$ siècle.

\section{La première génération de mutualistes : solidarité, entraide et démocratie}

La mutualité canadienne-française fut introduite à Ottawa durant les années 1860 par l'intermédiaire des réseaux migratoires. Les nouveaux arrivants, qui avaient l'expérience des sociétés de secours mutuel du Québec, cherchèrent à reconstituer les institutions du territoire d'origine en établissant l'Union Saint-Joseph d'Ottawa en 1863. Les deux initiateurs du projet, Léonard Desmarais et JeanBaptiste Champoux, ainsi que Cuthbert Bordeleau et Jean-Baptiste Ethier, qui se joignirent au groupe lors des premières rencontres, avaient contribué à la fondation et à l'incorporation de l'Union Saint-Joseph de l'Industrie de Joliette en 1861. Il est possible que d'autres membres fondateurs aient également acquis une certaine culture mutualiste dans le cadre des activités de différentes sociétés de secours mutuel québécoises. Ce noyau avait un objectif précis, soit de fonder une société de secours mutuel à l'image de celles qui existaient alors au Québec, en suivant le modèle de l'Union SaintJoseph de Montréal'. Comme ils l'avaient fait à Joliette, les fondateurs

7 Léonard Desmarais, Jean-Baptiste Champoux, Cuthbert Bordeleau, auxquels Jean-Baptiste Ethier s'ajouta lors de la cinquième rencontre, avaient tous participé à la fondation de l'Union Saint-Joseph de l'Industrie de Joliette. L'idée avait été lancée par Louis Mercil, un carrossier et ancien membre de l'Union Saint-Joseph de Montréal. Charles Leclerc, L'Union Saint-Joseph du Canada : son histoire, son cuvre, ses artisans!, Ottawa, Union Saint-Joseph du Canada, 1939, p. 11-12 ; Canada. Legislative Assembly, Parliament, 24 Vict., (1861), Bill: An Act to incorporate the Union of St. Joseph de l'Industrie, Québec, Stewart Derbishire et George Desbarats, Queen's Printer, p. 350-351; Petitclerc, "Nous protégeons l'infortune ", p. 52. 
utilisèrent la constitution et les règlements de l'organisation montréalaise pour mettre l'association sur pied et faciliter son établissement ${ }^{8}$.

Cette récupération incorpora de facto la méthode post mortem à l'Union Saint-Joseph d'Ottawa. Ce type de gestion imposait des tarifs égaux pour tous les membres, peu importe leur âge. À la cotisation des membres, s'ajoutait une contribution payable après le décès d'un sociétaire pour indemniser sa veuve ou sa famille? . L'absence de calcul pour déterminer les cotisations ne signifiait pas une ignorance du danger que pouvait entraîner l'émission d'un certificat à un aspirant qui représentait une incertitude quant à ses bonnes mœurs ou à sa santé. La gestion du risque résidait dans le processus d'admission. Le candidat devait satisfaire aux examens médicaux, connaitre deux membres actifs depuis au moins deux ans, répondre aux exigences du comité d'enquête et, finalement, être soumis à un ballottage secret qui décidait de son sort. L'établissement, en 1865 , de frais d'admission doublés pour les aspirants âgés de 40 à 55 ans, c'est-à-dire l'âge limite pour être admis, représentait la seule initiative financière de calcul du risque ${ }^{10}$. Elle incitait les gens à adhérer jeune et à continuer à payer les cotisations. Autrement, les exigences mensuelles et post mortem identiques pour tous les membres subsistèrent au sein de l'Union Saint-Joseph de sa fondation jusqu'en 1898. Les tarifs changèrent

\footnotetext{
${ }^{8}$ Assemblées générales et procès-verbaux, 22 mars 1863 au 6 septembre 1870, 6 avril 1863, Centre de recherche en civilisation canadienne-française (CRCCF), Fonds Union du Canada, C20, vol. 7.

9 Les cotisations et les indemnités varièrent considérablement entre 1863 et 1895. Entre 1870 et 1885 , la veuve recevait $300 \$$ au décès de son mari ; les paiements étaient de $0,50 \$$ au décès d'un membre et de $0,25 \$$ par mois pour les indemnités de maladie. En 1884, l'indemnité remise aux veuves augmenta à $400 \$$ et à $500 \$$ en 1887, grâce à la croissance des revenus de l'association. Cependant, les difficultés économiques obligèrent l'instauration d'un tarif administratif fixe de 0,75 \$ par mois à partir de 1893. Plusieurs paiements obligatoires et optionnels s'ajoutèrent aussi pour former des caisses spéciales, par exemple, pour remettre une somme d'argent à un membre lors du décès de sa femme ou encore pour offrir des bourses d'études aux orphelins. Leclerc, L'Union Saint-Joseph du Canada, p. 15-17.

${ }^{10}$ Constitution et règlements de l'Union Saint-Joseph d'Ottawa, 1887, p. 9, 12, 13, 24, 39, CRCCF, C20/B1/1.
} 
à quelques reprises au cours de cette période, mais demeurèrent les mêmes pour tous.

Cette forme d'égalitarisme, issue du modèle montréalais, s'enracinait dans les règles de l'organisation et déterminait les procédures des réunions. La codification dans la constitution et les règlements du déroulement de ces dernières assurait la pérennité de la structure organisationnelle en protégeant les valeurs fondatrices. D'abord, la participation obligatoire aux assemblées encourageait la sociabilité entre les membres. Ces derniers pouvaient simplement assister aux activités s'ils ne désiraient pas œuvrer activement à la gestion des affaires. À l'inverse, la structure offrait la possibilité pour tous les sociétaires d'être élus à un poste important. Des élections avaient lieu tous les six mois, afin d'empêcher qu'un petit groupe ne prenne le contrôle des affaires de l'association ${ }^{11}$. Le temps de parole était aussi limité afin de permettre au plus grand nombre de s'exprimer. Si le président ou les responsables de l'administration désiraient participer au processus décisionnel en émettant des opinions personnelles, ils devaient se retirer temporairement de leur poste pour s'adresser à la salle. Afin d'assurer la participation du plus grand nombre aux assemblées et aux funérailles d'un sociétaire, les absences entraînaient automatiquement des amendes. Cette méthode servait non seulement de mode de financement, mais elle était aussi une mesure coercitive dont le but était de favoriser la création d'un sentiment d'appartenance en rendant obligatoire la présence des membres aux activités ${ }^{12}$.

11 Entre la fondation et 1895 , seulement trois présidents siégèrent quatre semestres consécutifs, alors que la plupart ne demeuraient jamais en place plus d'un an. Constitution et règlements de l'Union Saint-Joseph d'Ottawa, 1887, p. 36, CRCCF, C20/B1/1 ; Liste des présidents depuis la fondation de la société le 22 mars 1863, CRCCF, C20/B3/3.

12 La démocratie était protégée par un protocole strict, inscrit dans les règlements, qui expliquaient en détail comment les réunions devaient se dérouler. La conduite des membres lors des réunions était aussi dictée par les règlements. Par exemple, il n'était pas possible d'intervenir plus de deux fois sur une même motion et de parler plus de dix minutes. Les intervenants devaient s'adresser directement au président. En cas d'impolitesse, la personne en défaut perdait son droit de parole 
Cette récupération organisationnelle établit une société de secours mutuel à Ottawa à l'image de sa consœur montréalaise. Les deux associations s'appuyaient cependant sur des fondements différents. L'élément déclencheur ou la situation de crise qui mena à la fondation de l'Union Saint-Joseph de Montréal découlait de la misère des ouvriers qui a suivi l'essor du capitalisme industriel, incarnée dans le cas d'Adolphe Guilbault. Ce dernier, un tailleur de pierre de Montréal, mourut en 1851 en laissant sa famille dans une condition financière précaire. Ses confrères de travail, qui se cotisèrent pour aider sa veuve, décidèrent de fonder une association mutuelle, l'Union Saint-Joseph de Montréal, pour éviter que ce genre de situation ne se reproduise ${ }^{13}$. Ainsi, comme le souligne Petitclerc, l'idéologie à la base du mouvement prit ses racines dans la volonté de la classe ouvrière, non pas uniquement d'établir une association de prévoyance, mais aussi d'offrir des ressources sociales en créant ce qu'il appelle une " famille fictive ", dont l'entraide mutuelle serait l'élément clé pour faire contrepoids à l'individualisme qui caractérisait cette ère de libéralisme ${ }^{14}$.

Dans le cas de l'Union Saint-Joseph d'Ottawa, il n'existait aucune base associative pour appuyer sa fondation. Outre les membres fondateurs, convaincus par leurs expériences antérieures des bienfaits que ce type d'association pouvait avoir pour les Canadiens français d'Ottawa, le projet reçut peu d'appui durant les deux premières années. La population ne sembla pas enthousiaste à y participer. Il

pour trois mois. Le fonctionnement de l'Union Saint-Joseph d'Ottawa ressemble en tout point à celui de l'Union Saint-Joseph de Montréal tel que décrit par Petitclerc dans son argumentation sur le caractère démocratique des sociétés de secours mutuel de première génération. Petitclerc, "Nous protégeons l'infortune ", p. 71-78; Constitution et règlements de l'Union Saint-Joseph d'Ottawa, 1887, p. 12, CRCCF, C20/B1/1.

13 Jacques A. Plinguet, Souvenirs sur les commencements de l'Union St. Joseph de Montréal, Montréal, Des Presses à vapeur de Plinguet \& Laplante, 1866, p. 1-2.

14 Concernant l'Union Saint-Joseph d'Ottawa, il n'existe aucune preuve d'une solidarité réelle entre les membres ; c'est-à-dire qu'elle existe seulement dans la mesure où la constitution et les règlements l'obligent, mais les actions ne dépassent jamais le cadre financier. Petitclerc, "Nous protégeons l'infortune », p. 57-63. 
fallut d'ailleurs quelques années pour voir un nombre suffisant de Canadiens français se joindre à l'association afin d'assurer la viabilité du projet. La majorité des sociétaires qui joignirent l'Union SaintJoseph d'Ottawa n'avait aucune expérience de la mutualité. Conséquemment, la culture mutualiste qui prit forme put se définir selon la réalité sociale des Canadiens français d'Ottawa. Son développement fut ainsi marqué au cours des premières décennies par le contexte évolutif difficile des Canadiens français à Ottawa. Le caractère national du phénomène associatif, alors que chaque groupe possédait ses propres institutions, obligeait les Canadiens français à renforcer leur position institutionnelle durant les années 1850 et 1860 pour assurer leur survie en tant que groupe.

Comme l'explique Robert Choquette, l'Église catholique et ses institutions formaient l'assise de la communauté canadienne-française d'Ottawa au XIX ${ }^{e}$ siècle. Le clergé jouait un rôle clé dans la survivance du groupe pendant cette période d'enracinement dans les conditions difficiles d'évolution causées par les rivalités ethnolinguistiques ${ }^{15}$. Son appui était nécessaire pour garantir la viabilité du projet. Les relations entre les mutualistes et le clergé catholique durant la décennie précédente n'avaient pas toujours été faciles. L'Union Saint-Joseph de Montréal avait dû se soumettre, en 1857 , à la visite d'un chapelain aux réunions, afin que ce dernier s'assure de la moralité de l'association. Cette situation ne fut pas sans créer un certain émoi au sein des membres, qui refusaient généralement toute forme d'ingérence. Au terme d'une querelle qui a duré quatre ans, le clergé obligea la tenue d'un vote pour imposer la présence du chapelain aux réunions. Il est possible que les membres fondateurs de l'Union Saint-Joseph d'Ottawa aient voulu éviter une situation semblable à celle qui s'était déroulée

15 Ces thèmes sont abordés en profondeur dans les travaux de Robert Choquette. Ce dernier met l'accent sur les rivalités entre Canadiens français et Irlandais au sein du clergé, et le rôle de l'Église dans la sauvegarde de la langue française par son rôle dans le domaine de l'éducation. Robert Choquette, L'Église catholique dans l'Ontario français du dix-neuvième siècle, Ottawa, Éditions de l'Université d'Ottawa, 1984, p. 140-153, 223-245, 281-312. 
à Montréal. En somme, l'Union Saint-Joseph d'Ottawa se montra beaucoup plus ouverte au clergé, et une relation cordiale s'établit dès la fondation. Lors des premières réunions, les membres fondateurs demandèrent la présence d'un chapelain, qu'ils élurent symboliquement à l'unanimité. Les privilèges qui lui étaient accordés dépassaient ceux consentis au chapelain de l'Union Saint-Joseph de Montréal. L'association accorda le droit de parole au chapelain en 1865 pour discuter de questions morales et religieuses. Ces privilèges dépassaient cependant la sphère morale. Le chapelain Routhier mentionna, d'ailleurs, lors d'une assemblée en $1885:$ : Je voudrais continuer à donner quelques conseils pratiques au sujet de l'économie et faire des membres de l'Union Saint-Joseph des hommes sérieux et de bons chrétiens ${ }^{16}$." Le rôle temporel du clergé au sein de l'Union Saint-Joseph est difficilement qualifiable à cause du manque de sources jusqu'aux années 1880 .

L'Union Saint-Joseph d'Ottawa joua un rôle institutionnel important en étant la seule société de secours mutuel canadiennefrançaise de la ville jusqu'au milieu des années 1870 . De plus, puisque $94 \%$ des Canadiens français habitaient le quartier de la Basse-Ville en 1871, des gens de la classe populaire pour la plupart, l'identité associative pouvait s'appuyer sur ces liens communautaires pendant les deux premières décennies ${ }^{17}$. C'est dans ce quartier que l'Union Saint-Joseph avait ses bureaux et tenait ses réunions. La plupart des hommes de la Basse-Ville pouvaient participer à ses activités, à l'exception des gens de professions libérales. Cette interdiction visait à éviter qu'une élite instruite dirige la destinée de l'association. Ils étaient peu nombreux à subir cette restriction puisque les Canadiens français plus aisés habitaient le quartier de la Haute-Ville. Malgré cette importance en tant que seule association de prévoyance

${ }^{16}$ Procès Verbaux, 3 novembre 1885 au 26 juillet 1887, 3 novembre 1885, CRCCF, C20, vol. 10.

17 Fernand Ouellet, "Disparités socio-économiques et culturelles à Ottawa en 1871 ", Cahiers Charlevoix : études franco-ontariennes, vol. 6, Sudbury, Prise de parole, 2004, p. 23. 
canadienne-française, elle conserva un caractère apolitique durant les deux premières décennies de son histoire. La constitution et les règlements repris du modèle montréalais interdisaient toute action hors de la sphère économique qui aurait permis l'adoption d'un projet social différent. Les activités se limitaient au secours de la classe populaire et aux objectifs de sociabilité, sans possibilité de prise de position officielle sur des sujets nationaux marquants. Elle n'était pas neutre pour autant par son intégration à ce réseau institutionnel canadien-français émergent. Son rôle demeurait important dans la lutte contre l'assimilation par l'offre d'un service de prévoyance en français. L'Union Saint-Joseph participa activement à la sauvegarde de la culture canadienne-française, surtout à partir des années 1880 . L'achat de son édifice dans la Basse-Ville, à l'angle des rues York et Dalhousie, en 1884, contribua à ce développement. Par exemple, après l'incendie d'une école catholique, l'organisation accepta de prêter sa grande salle gratuitement pour servir temporairement de classe $^{18}$. Cette salle fut sollicitée par bon nombre d'organisations pour y tenir leur assemblée hebdomadaire : la Société Saint-Pierre, l'Union Saint-Thomas, la Société Saint-Louis, l'Association mutuelle catholique, et plusieurs associations paroissiales ${ }^{19}$. En plus d'assurer un certain revenu à l'Union Saint-Joseph, sa location permettait de conserver des liens privilégiés avec les organisations mutuelles d'Ottawa. Cet édifice lui donnait une visibilité et facilitait son engagement social, ce qui avait un effet positif sur le recrutement.

La conscience identitaire de l'association fut également marquée durant les années 1880 par la participation de ses membres à de grands rassemblements patriotiques. Quelques liens interorganisationnels s'étaient formés par le partage de constitutions et de règlements, et par l'admission d'anciens membres de mutuelles

18 Assemblée générale, livre de minutes n 1, 2 août 1887 au 25 octobre 1892, 31 janvier 1888, CRCCF, C20, vol. 11.

19 Procès Verbaux, 2 mai 1882 au 27 octobre 1885, 8 septembre 1885, 24 septembre 1885, CRCCF, C20, vol. 9 ; Procès Verbaux, 3 novembre 1885 au 26 juillet 1887, 3 novembre 1885, CRCCF, C20, vol. 10. 
québécoises $^{20}$. Ces interactions prirent une autre forme à partir des années 1880 alors que se tinrent de grands congrès pour rassembler les Canadiens français du territoire d'origine et ceux de la diaspora. Les sociétés de secours mutuel y participèrent en organisant des rencontres dans le cadre de ces rassemblements. Par exemple, l'Union Saint-Joseph envoya des délégués en 1880 à une réunion des sociétés de bienfaisance qui se déroula à Québec en marge des fêtes de la Saint-Jean-Baptiste ${ }^{21}$. En 1885, ceux-ci se rendirent à West Farnham, dans le cadre d'un grand rassemblement de sociétés de secours mutuel francophones catholiques, réunissant des associations canadiennes et américaines pour fêter et discuter de mutualité ${ }^{2}$. Parfois, ces événements prenaient une ampleur considérable comme ce fut le cas lors du $25^{\mathrm{e}}$ anniversaire de l'Union Saint-Joseph d'Ottawa en 1888. Le congrès et les festivités regroupèrent au moins quinze sociétés de secours mutuel comptant plusieurs dizaines de sociétaires chacune, dont ceux de l'Union Saint-Joseph de Saint-Hyacinthe, de Farnham, de Sherbrooke, de Montréal, de Saint-Sauveur, de Saint-Roch-deQuébec, de Lévis, de Notre-Dame-de-Beauport et de plusieurs autres. Les délégations les plus nombreuses furent celles de l'Union SaintJoseph d'Ottawa, de l'Union Saint-Joseph de Hull avec 300 membres, et de l'Union Saint-Joseph de Farnham, avec plus de 100 sociétaires. Les défilés, les banquets, les courses de chevaux ainsi que les autres activités furent l'occasion de tisser des liens solides entre les différentes sociétés de secours mutuel locales canadiennes-françaises catholiques provenant de diverses villes ${ }^{23}$. Des relations et une correspondance se prolongèrent au-delà de ces rencontres, donnant souvent lieux à

${ }^{20}$ Le président de l'Union Saint-Joseph de Montréal assista à une assemblée de l'Union Saint-Joseph d'Ottawa, et les procès-verbaux font mention de plusieurs lettres reçues par l'organisation montréalaise. Assemblée générale, Procès verbaux, 7 mai 1878 au 25 avril 1882, 28 janvier 1879, CRCCF, C20, vol. 8.

${ }^{21}$ Assemblée générale, Procès verbaux, 7 mai 1878 au 25 avril 1882, 7 décembre 1880, 1 juin 1880, CRCCF, C20, vol. 8.

22 Procès Verbaux, 3 novembre 1885 au 26 juillet 1887, 3 novembre 1885, CRCCF, C20, vol. 10.

23 Assemblée générale, Livre de minutes n 1,2 août 1887 au 25 octobre 1892, 12 juin 1888, CRCCF, C20, vol. 11. 
des visites ${ }^{24}$. Le développement de cette conscience nationaliste au sein de l'Union Saint-Joseph influença l'association dans la quête d'une solution pour parer la crise qui affectait la mutualité durant les années 1890. La solidarité nationale devenait graduellement une base solide et acceptable pour y appuyer la mutualité, surtout pour les Canadiens français qui évoluaient en milieu minoritaire.

\section{La transformation du modèle originel}

Le fonctionnement démocratique de la première génération de mutuelles permettait une adaptation de la structure administrative au contexte évolutif de ses membres. Les changements dépendaient des influences locales et des mutations de la doctrine mutualiste dominante, que ce soit par une modification à la législation ou le développement de nouvelles formes de mutualité. Toutefois, selon le sociologue Albert Meister, ces vagues de changements étaient inévitables. Son échelle d'évolution des coopératives montre que ces associations devaient entrer graduellement dans une deuxième phase de développement, où l'économie primerait les objectifs d'entraide et de solidarité. Puisque l'initiative coopérative se voulait une tentative visant à contrebalancer les effets pervers du capitalisme, l'enthousiasme des premiers jours pour le projet social tendait généralement à surpasser les considérations économiques. Cependant, la croissance d'une association de ce genre se butait inévitablement à certaines contraintes financières, obligeant à reconsidérer les objectifs de départ à cause de son manque de compétitivité par rapport aux entreprises privées offrant les mêmes services ${ }^{25}$. Les causes menant à cette rupture avec le plan original pouvaient varier d'une organisation à l'autre. Dans le cas de l'Union Saint-Joseph, les expériences des années 1880 et 1890 , c'est-à-dire la concurrence des sociétés fraternelles, amenèrent

${ }^{24}$ Les procès-verbaux notent l'envoi de lettres pour des invitations diverses, souvent pour venir " chômer " la fête patronale, à partir des années 1880 .

25 Albert Meister, La participation dans les associations, Paris, Les éditions ouvrières, 1974, p. 192 à 212 . 
une certaine transition, laquelle entraîna la reconceptualisation de son idéal mutualiste. Sa croissance interne, la concurrence entre les organisations et les difficultés économiques conduisirent à la refonte de ses méthodes actuarielles et de son administration. Conséquemment, les bases idéologiques qui amenaient les membres à participer à ses activités s'en trouvèrent complètement modifiées.

Les premières contraintes du modèle organisationnel de l'Union Saint-Joseph se firent sentir au cours des années 1880. Cette décennie marqua un tournant pour l'Union, stimulée par une croissance sans précédent de ses effectifs. Profitant de l'augmentation de la population canadienne-française de la ville, de l'amélioration des conditions économiques ainsi que d'un engouement social grandissant pour la mutualité, le nombre de sociétaires passa de 269 à 794 entre 1880 et $1888^{26}$. Conséquemment, la participation souffrit de cette croissance trop rapide. La possibilité d'intervention lors des débats diminua considérablement, et le processus décisionnel fut alourdi par l'augmentation des intervenants. Par la même occasion, les réunions devenaient une occasion de sociabilité, où certains membres préféraient jouer aux cartes plutôt que de participer activement aux discussions, conséquence de la diminution du pouvoir d'intervention ${ }^{27}$.

Cette croissance de l'Union Saint-Joseph correspond à une période faste pour la mutualité canadienne-française à Ottawa. Le mouvement se transformait et profitait d'un engouement pour la prévoyance. Depuis les années 1870 , de nouvelles sociétés de secours

${ }^{26}$ Pour être admis, un aspirant devait résider dans la ville depuis au moins deux ans, en plus d'obtenir l'approbation de membres de l'organisation qui le connaissaient personnellement depuis cette période. Ces données sont datées du 30 avril. Rapports semestriels ajoutés aux procès-verbaux des réunions, Assemblée générale, procès verbaux, 7 mai 1878 au 25 avril 1882, CRCCF, C20, vol. 8 ; Assemblées générales, Livre de minutes $\mathrm{n}^{\circ} 1,2$ août 1887 au 25 octobre 1892, CRCCF, C20, vol. 10.

27 Procès Verbaux, 3 novembre 1885 au 26 juillet 1887, 1 mars 1887, CRCCF, C20, vol. 10 ; Séance extraordinaire du [?] juin 1887, 3 novembre 1885 au 26 juillet 1887, CRCCF, C20, vol. 10 ; Assemblées générales, Livre de minutes $\mathrm{n}^{\circ} 1,2$ août 1887 au 25 octobre 1892, 16 juillet 1889, CRCCF, C20, vol. 11. 
mutuel locales s'établissaient pour combler différents besoins non couverts par les associations existantes. C'est dans ce but que fut fondée l'Union Saint-Thomas en 1875, dont l'adhésion s'adressait à l'ensemble de la population canadienne-française, sans distinction de classe. D'autres associations mutualistes canadiennes-françaises furent établies, dont la Société Saint-Pierre en 1873, la Société SaintAntoine de Padoue en 1885 et l'Union Saint-Jean-Baptiste en 1887. Cette multiplication des options de prévoyance eut toutefois pour effet d'augmenter la concurrence ${ }^{28}$. Cependant, aucune de ces mutuelles ne réussit à obtenir autant de succès que l'Union Saint-Joseph. La deuxième plus populaire, l'Union Saint-Thomas, comptait 489 membres en 1893 alors que les effectifs de l'Union Saint-Joseph se chiffraient à $740^{29}$. Cependant, la présence d'un trop grand nombre d'associations menaçait la stabilité de chacune d'entre elles. La méthode de gestion financière utilisée par les sociétés de secours mutuel locales nécessitait un recrutement constant de nouveaux aspirants pour assurer les revenus nécessaires au paiement des indemnités. L'Union Saint-Joseph vit, d'ailleurs, une légère diminution de ses sociétaires au début des années 1890 .

De plus, un nouveau type de mutualité gagna en popularité durant les années 1880 : les sociétés fraternelles. Ces grandes sociétés de secours mutuel avaient profité de la croissance rapide d'Ottawa pour y implanter des succursales ${ }^{30}$. L'Ancient Order of United Workmen, les Forestiers catholiques, la Catholic Mutual Benefit Association, le Canadian Order of Foresters et d'autres associations commencèrent à desservir la population d'Ottawa pendant la décennie. Il est difficile d'évaluer le nombre exact de membres au

${ }_{28}$ Ontario. Ontario Sessional Papers, Report of the Inspector of Insurance and Registrar of Friendly Societies (1893), Toronto, Queen's Printer, 1895, vol. XXVII, part III, p. C85-C90, C124-C127.

29 Ibid., p. C85-C90, C124.

30 Durant les années 1870 , le mouvement associatif anglophone d'Ottawa était dominé par les ordres maçonniques et orangistes. La mutualité y était souvent optionnelle. 
sein des succursales de ces associations étrangères puisqu'elles n'ont presque pas laissé de traces ${ }^{31}$. En l'absence de sociétés fraternelles canadiennes-françaises à Ottawa durant les années 1880, les Canadiens français semblent s'être tournés vers les associations catholiques sans restriction linguistique qui s'implantèrent à Ottawa pendant la deuxième moitié des années $1880^{32}$. De plus, un certain nombre de Canadiens français participaient aux activités des associations non confessionnelles. Il n'existe pas de données précises à ce sujet, mais les administrateurs de l'Union Saint-Joseph se plaignaient de voir certains compatriotes adhérer à ces sociétés ${ }^{33}$. L'arrivée de plusieurs conseils locaux de grandes associations fraternelles étrangères menaçait les petites mutuelles canadiennes-françaises, dont l'Union SaintJoseph, principalement en offrant des services beaucoup plus variés et une plus grande stabilité financière. Les sociétés nationales et internationales, qui implantèrent des bureaux de perception un peu partout pour augmenter leur bassin de recrutement, nuisaient sérieusement aux sociétés de secours mutuel déjà en place.

La concurrence devint un problème, surtout au début des années 1890. L'instabilité provenait principalement de la crise économique de 1891 qui toucha le commerce du bois, principale industrie de la région outaouaise. La capacité des Canadiens français de payer les

31 Les annuaires des villes nous donnent une idée générale, mais parfois le classement des associations francophones porte à confusion, en plus d'être souvent incomplet.

32 Assemblée générale, Livre de minutes $\mathrm{n}^{\circ} 1,2$ août 1887 au 25 octobre 1892, 3 novembre 1891, CRCCF, C20, vol. 11 ; Grimard et Vallières, Travailleurs et gens d'affaires canadiens-français en Ontario, p. 190 ; Catholic Mutual Benefit Association of Canada, C.M.B.A. Herald, 22 juillet 1891, Kingston, p. 8 ; The Ottawa Directory, vol. 11, 1884-1885, p. 304 ; The Ottawa Directory: 1891-1892, p. 36.

33 Par exemple, en 1894, un comité fut mis sur pied pour étudier comment l'Union Saint-Joseph pourrait offrir autant de services que les associations étrangères puisqu'elles semblaient attirer un nombre grandissant de membres. Assemblées générales, Minute book, 1 novembre 1892 au 6 décembre 1897, 13 novembre 1894, CRCCF, C20, vol. 12. 
cotisations s'en trouva considérablement diminuée ${ }^{34}$. Les répercussions sur l'Union Saint-Joseph furent immédiates, alors que le nombre de membres passa de 760 en 1892 à 723 en 1894, devant l'incapacité d'admettre suffisamment de candidats pour combler les radiations et les décès ${ }^{35}$. Cette difficulté à se renouveler risquait d'augmenter à long terme les moyennes d'âge et les taux de mortalité, rendant ainsi la mutuelle beaucoup moins attrayante. La diminution du nombre de cotisants pouvait mener à une baisse des indemnités remises aux veuves, à une période où les sociétés fraternelles garantissaient le montant de leurs certificats d'assurance ${ }^{36}$. Cette difficulté favorisait les grandes mutuelles catholiques, dont les succursales nouvellement installées à Ottawa recrutaient de plus en plus d'aspirants.

La crise que connut l'Union Saint-Joseph d'Ottawa au début des années 1890 devint l'élément déclencheur qui a mené au changement de son modèle administratif. S'ils s'étaient montrés inefficaces durant les années 1880 pour répondre aux objectifs de solidarité et d'entraide à cause de la lourdeur du processus décisionnel, la constitution et les règlements protégeaient les fondements de l'organisme en rendant obligatoire la participation des sociétaires. Ainsi, devant la présence de l'ensemble du groupe aux réunions, les tentatives de révision se butèrent, en 1887, aux réticences d'un nombre important de membres à voir leur association se transformer, ce qui engendrait de longs débats ${ }^{37}$. Le modèle participatif s'enfonçait dans ce cercle vicieux, incapable de se réformer. Pour que le processus décisionnel

${ }^{34}$ Jean Hamelin et Yves Roby, Histoire économique du Québec, 1851-1896, Montréal, Fides, 1971, p. 95.

35 Ontario. Ontario Sessional Papers, Report of the Inspector of Insurance and Registrar of Friendly Societies (1893), vol. XXVII, part III, p. C85-C90 ; Ontario. Ontario Sessional Papers, Report of the Inspector of Insurance and Registrar of Friendly Societies (1894), Toronto, Queen's Printer, 1896, vol. XXVIII, part II, p. C78-C81.

36 L'Union Saint-Joseph garantissait également le montant de l'indemnité de la veuve, mais un changement constitutionnel suffisait à modifier la valeur du certificat.

37 Procès Verbaux, 3 novembre 1885 au 26 juillet 1887, $1^{\text {er }}$ mars 1887, CRCCF, C20, vol. 10 . 
puisse fonctionner adéquatement, moins de gens devaient participer aux délibérations. Il n'existait, toutefois, aucun moyen efficace pour réduire le pouvoir des sociétaires. La participation demeurait, malgré les difficultés, un élément clé du lien d'appartenance qui unissait l'Union Saint-Joseph et ses membres ; sa révocation aurait menacé la dynamique qui avait nourri l'organisation depuis sa fondation. Les sociétaires devaient maintenant choisir entre la conservation des objectifs originels et risquer la faillite, ou entamer des réformes qui pouvaient mener à la transformation du projet social de l'Union Saint-Joseph.

La réforme n'aurait pas nécessairement amené une refonte du projet social dans sa totalité, n'eût été l'intervention du gouvernement pour régir les certificats d'assurance des sociétés de secours mutuel. La nouvelle loi ontarienne de 1892, adoptée sous le nom d'Act respecting Insurance Corporations, donna le coup de grâce au modèle organisationnel initial. Stimulé par l'Enquête sur les rapports qui existent entre le capital et le travail au Canada, qui démontra l'importance de la mutualité pour la classe ouvrière, le gouvernement ontarien décida d'intervenir pour assurer une plus grande stabilité des sociétés de secours mutuel ${ }^{38}$. Ainsi, celles qui, comme l'Union Saint-Joseph, garantissaient des indemnités fixes aux veuves reçurent une reconnaissance de leurs certificats d'assurance au même titre que les compagnies privées. Ce nouveau statut transforma la dynamique interne de l'Union Saint-Joseph, en interdisant l'imposition d'amende pour la non-participation aux activités obligatoires. En vertu de cette loi, le coût des certificats d'assurance, ce qui incluait les paiements divers tels que les amendes, devait être égal ou déterminé par l'âge et le risque au sein d'une même association. Le gouvernement retirait

38 Canada. Commission royale d'enquête sur les relations entre le capital et le travail, Enquête sur les rapports qui existent entre le capital et le travail au Canada: province d'Ontario, Ottawa, A. Senecal, 1889, vol. 6, p. 159-160 ; Ontario. Ontario Sessional Papers, The Report of the Inspector of Insurance (1894), 59 Vict., vol. XXVIII, part II, n 10, Toronto, Lud K. Cameron, Queen's Printer, 1896, p. C180. 
ainsi à l'Union Saint-Joseph et aux autres sociétés de secours mutuel la mesure coercitive qui permettait la survie de la sociabilité et du modèle participatif ${ }^{39}$.

\section{La transition vers une société nationale}

Ces contraintes législatives, économiques et organisationnelles mirent fin au projet social initial de l'Union Saint-Joseph. L'incapacité de s'appuyer sur des mesures coercitives pour assurer une sociabilité et une entraide entre les membres obligea l'association à trouver d'autres bases pour justifier ses activités. La première solution proposée à la crise fut de tenter une fusion des mutuelles canadiennes-françaises d'Ottawa sous la bannière de l'Union Saint-Joseph afin de créer une seule grande association canadienne-française dans la ville. La tentative échoua pour des raisons économiques et idéologiques. Les mutations financières qu'avaient connues les sociétés de secours mutuel lors des dernières décennies compliquaient l'harmonisation des caisses d'assurances ainsi que la transition vers une gestion uniforme $e^{40}$. Puisque l'Union Saint-Joseph demeurait la plus puissante des cinq mutuelles canadiennes-françaises établies à Ottawa, l'entreprise visait davantage à les absorber qu'à créer une fédération quelconque. Les sociétaires des autres associations refusèrent l'abandon de leur autonomie et, par la même occasion, du projet social pour lequel ils

39 Par cette loi, les contrats des sociétés de secours mutuel étaient reconnus au même titre que ceux des compagnies d'assurances. En conséquence, elles étaient tenues de s'enregistrer et d'obtenir un permis pour offrir des services d'assurances dans la province. Elles devaient présenter deux copies de leur constitution et règles, ainsi que tous les détails concernant leur fonctionnement afin de recevoir ce privilège. Annuellement, les sociétés de secours mutuel devaient remettre un rapport financier au registraire. Ces rapports étaient rendus publics afin que les membres puissent s'assurer de l'état de leur association, suivant ainsi les recommandations de la commission d'enquête. William H. Hunter, The Insurance Corporation Act, 1892, Toronto, Carswell, 1893, p. 77, 169, 171 ; Ontario. Statutes of Ontario, 55 Vict., (1892), An Act respecting Insurance Corporations, Toronto, Lud K. Cameron, Queen's Printer, 1892, p. 195, 204.

${ }^{40}$ Les indemnités et les cotisations des associations différaient trop pour permettre une consolidation. 
avaient œuvré pendant des années. Finalement, seule l'Union SaintPierre fut annexée en 1895. L'initiative s'avéra insuffisante pour assurer la stabilité financière de l'organisation puisqu'elle n'améliorait pas le statut de l'Union Saint-Joseph par rapport aux sociétés fraternelles, qui continuaient leur ascension.

La solution à la crise devait passer par la refonte de l'administration, ce qui nécessitait certaines connaissances du domaine actuariel dépassant le niveau d'éducation de la plupart des sociétaires. L'interdiction d'admettre des aspirants issus des professions libérales restreignait le nombre de gens instruits dans les rangs de l'organisme. D'ailleurs, jusqu'à la transformation administrative de 1895, qui ouvrit finalement la porte de l'Union Saint-Joseph à tous les hommes canadiensfrançais, les sociétaires acceptèrent difficilement l'aide extérieure. Ils refusèrent même l'aide de Napoléon-Antoine Belcourt pour résoudre la crise du financement, alors que ce dernier agissait à titre d'avocat de l'organisation ${ }^{41}$. Cependant, certains membres, sociétaires depuis très longtemps, prospérèrent et acquirent certaines connaissances clés qui ont facilité la quête d'une solution. Ceux-ci formèrent le noyau à la base des réformes. C'est le cas d'Olivier Durocher, admis comme simple cordonnier en 1866 et qui fut maire d'Ottawa en 1892-1893. Ce dernier présidait l'organisation lors des transformations administratives et actuarielles de 1895 et de $1898^{42}$. D'autres réformateurs avaient l'expérience de l'administration au sein des grandes sociétés fraternelles. M. J. N. Rattey, premier vice-président après la réforme, était chef de bureau chez M. Belcourt et Ritchie ${ }^{43}$. À son curriculum s'ajoutaient les titres "d'auditeur " de la Société des artisans et de grand député de la Catholic Mutual Benefit Association ${ }^{44}$. L'exemple de Charles

41 Napoléon-Antoine Belcourt n'avait pas encore été élu au Parlement à ce moment, mais il travaillait à la Faculté de droit de l'Université d'Ottawa. Assemblée générale, Livre de minutes $\mathrm{n}^{\circ} 1,2$ août 1887 au 25 octobre 1892, 6 mai 1890, CRCCF, C20, vol. 11.

42 L'Union Saint-Joseph d'Ottawa, Le Prévoyant, n ${ }^{\circ} 28,15$ mars 1901, p. 6.

43 Il s'agit du cabinet de Napoléon-Antoine Belcourt.

44 L'Union Saint-Joseph d'Ottawa, Le Prévoyant, n 27, 15 février 1901, p. 14. 
Desjardins, directeur général de l'organisme au moment de la transformation de 1895, est aussi très éloquent. Après avoir terminé des études commerciales à Lévis, il s'installa à Ottawa en 1869 pour travailler comme télégraphiste. En 1874, il entreprit une carrière dans le domaine de l'assurance. Une dizaine d'années plus tard, il représentait plusieurs compagnies importantes, entre autres la Caledona Insurance Company of Edinburg, la Phoenix Insurance Company of London, la Northern Assurance co. of Aberdeen \& London et plusieurs autres ${ }^{45}$. La date de son admission au sein de l'Union SaintJoseph est inconnue, mais il œuvra comme président durant les deux semestres de 1885. Ses connaissances du domaine de l'assurance contribuèrent grandement à la formulation d'une solution à la crise actuarielle.

La crise permit à cette petite élite de vaincre les barrières constitutionnelles de l'association. Entre la fin des années 1880 et la transformation de 1895 , le modèle participatif alourdissait considérablement les discussions, empêchant la formation de débats concernant la modification des règlements. Puisque l'ensemble des décisions devait faire l'objet d'un vote, que ce soit l'attribution des indemnités ou l'admission des nouveaux membres, il y avait peu de temps pour discuter des amendements. Ce n'est qu'avec la gravité de la crise financière au début des années 1890 que la nécessité de modifier le fonctionnement de l'association entraîna des actions concrètes. Pour remédier à la situation, des comités furent nommés en 1890, 1893 et 1894 afin de proposer à l'assemblée des modifications aux règlements et au système de cotisation ${ }^{46}$. La principale lacune de l'organisation demeurait le financement des caisses servant à payer les indemnités. L'insolvabilité résultant des méthodes implantées lors des premières réunions commençait à peser lourd sur les finances à la suite du déclin

45 L'Union Saint-Joseph d'Ottawa, Le Prévoyant, n² 24, 15 novembre 1900, p. 43 ; The Ottawa Directory, A. S. Woodburn, 1885, p. i.

46 Assemblée générale, Livre de minutes n 1, 2 août 1887 au 25 octobre 1892, 6 mai 1890, CRCCF, C20, vol. 11 ; Assemblée générale, Minute book, 1 novembre 1892 au 6 décembre 1897, 9 mai 1893, 13 novembre 1894, CRCCF, C20, vol. 12. 
de ses effectifs au début des années 1890. Cependant, les membres n'auraient pas accepté facilement une augmentation des cotisations. Les réformes devaient reposer sur des bases sociales et financières acceptables pour les sociétaires.

Le comité nommé en 1894 proposa une nouvelle constitution, à accepter ou à refuser en bloc. Elle permettait à l'Union Saint-Joseph d'établir des succursales, d'implanter des bureaux de perception ou des conseils locaux afin d'augmenter son bassin de recrutement et diminuer l'impact de son instabilité financière en ajoutant des cotisants. La stratégie misait sur la bonne relation entre l'association et l'archevêché, car l'organisme espérait recevoir une aide du clergé locàl pour publiciser ses activités et fonder des succursales dans les différentes paroisses de l'archidiocèse d'Ottawa. Cette constitution permettait de conserver les cotisations uniformes sans augmenter le fardeau financier des cotisants. Les sociétaires acceptèrent à l'unanimité, en 1895, la constitution qui établissait une structure fédérale pour maintenir une assurance de valeur supérieure par rapport aux sommes versées. Les dirigeants du comité la présentèrent comme la seule solution pour éviter une hausse substantielle des cotisations, laissant même planer la possibilité d'une baisse si les effectifs venaient à augmenter ${ }^{47}$. La méthode post mortem, quant à elle, demeura en place jusqu'à la deuxième phase de la réforme, qui se déroula en 1898 .

Lacceptation de cette nouvelle constitution démontre à quel point l'économie personnelle des membres primait l'idéal social des premiers jours, surtout depuis la suppression des mesures coercitives relatives à la participation obligatoire des membres aux assemblées pour se conformer à la législation provinciale. Par l'établissement d'une structure fédérale et d'un comité central pour gérer l'ensemble des activités, les sociétaires abandonnèrent une partie de leur pouvoir décisionnel. Ils pouvaient toujours intervenir lors des congrès annuels et participer aux assemblées des conseils locaux, mais la participation

47 Constitution et règlements de l'Union Saint-Joseph d'Ottawa, A. Bureau \& Frères, 1895, p. 12-19, CRCCF, C20/B1/3. 
directe, qui constituait le fondement de la mutualité de la première génération, fut grandement diminuée et continua de l'être à mesure que les effectifs de l'association crûrent. Le pouvoir de la plupart des sociétaires se limitait à voter pour les délégués qui prenaient les décisions, ce qui ne leur permettait plus de jouer un véritable rôle ${ }^{48}$. La complexification de la gestion limitait indirectement l'accès aux postes administratifs aux gens ayant des connaissances de la mutualité. Il s'agissait du prix à payer pour conserver une assurance à bas prix.

Cette perte de contrôle des sociétaires contribua à la transition et à l'adoption de nouvelles réformes en 1898, qui visaient à établir une gestion des finances suivant des principes actuariels reconnus. Le système post mortem en place devint rapidement impossible à gérer avec la croissance du réseau de succursales. Puisque les membres étaient tenus de payer une seule prime de décès par mois, les retards dans la perception des cotisations atteignaient six mois, à l'été $1897^{49}$. Pour remédier à la situation, le comité fédéral proposa une nouvelle constitution, qui entra en vigueur en 1898. Celle-ci abolissait la méthode post mortem pour la remplacer par celle des primes graduées, soit l'établissement de paiements mensuels variables, établis selon l'âge lors de l'admission. Cette réforme instituait une gestion des finances influencée par les pratiques de l'assurance privée ${ }^{50}$.

La stratégie des administrateurs pour assurer l'acceptabilité de ces changements consista à proposer une grille de paiements inférieurs aux exigences déjà en place. Ils espéraient ainsi, une fois la réforme acceptée, augmenter le coût des certificats d'assurance pour les nouveaux membres de façon à leur faire payer l'insuffisance des cotisations des anciens membres. Pour la plupart des groupes d'âge, le plan prévoyait

48 Ibid., p. 2-3, 38-43.

49 L'Union Saint-Joseph d'Ottawa, Bulletin offciel, 15 octobre 1897, p. 87 ; L'Union Saint-Joseph d'Ottawa, Bulletin officiel, 15 novembre 1897, p. 95.

50 L'adoption de cette méthode differe des compagnies d'assurances, car les primes ne sont pas encore actuariellement solvables, c'est-à-dire que le membre continue de payer une cotisation inférieure à la valeur de son certificat d'assurance. 
que les versements mensuels passeraient de 1,25 \$à moins de $1 \$$. Cette mesure contribua fortement à l'acceptation des nouvelles politiques $^{51}$. D'ailleurs, ces faibles cotisations, insolvables d'un point de vue actuariel et insuffisantes pour assurer la viabilité des caisses, demeurèrent en place pendant quelques années en tant que méthode de propagande pour faciliter la multiplication des succursales. Les primes furent rétablies en 1900 à un niveau plus solvable pour les nouveaux membres ${ }^{52}$. Les dirigeants prévoyaient, de cette façon, absorber l'insolvabilité des sociétaires plus anciens, dont le nombre deviendrait négligeable grâce au recrutement et à l'implantation de succursales qui progressaient à un rythme soutenu.

La réforme de 1898 anéantit ce qui restait des valeurs initiales de l'Union Saint-Joseph. Elle établissait un calcul du risque basé sur l'individu, alors qu'il reposait plutôt sur l'ensemble du groupe jusqu'à cette date, par l'imposition de cotisations uniformes pour tous. Malgré le caractère radical de ce changement, peu de sociétaires s'opposèrent à cette réforme. Pour certains anciens membres, qui avaient contribué à la première génération de société de secours mutuel, la transition idéologique ne s'effectua pas facilement. Les opposants furent, toutefois, mal organisés pour lutter contre ces transformations exigées par l'élite mutualiste, qui jugeait archaïque l'ancienne forme de calcul du risque. D'autres facteurs expliquent, cependant, la facilité avec laquelle la majorité des sociétaires accepta les réformes. D'abord, l'Union SaintJoseph ne répondait plus aux exigences d'une partie de ses membres. La transformation du mouvement associatif au cours des années 1880 et 1890 avait contribué à développer un rapport de clientèle entre les sociétés de secours mutuel et leurs membres. La mutualité canadienne-française était née d'un besoin de solidarité urbaine au milieu du XIX ${ }^{e}$ siècle, causé par la rupture des liens ruraux. Son

51 Leclerc, L'Union Saint-Joseph du Canada, p. 15 à 17.

52 La solvabilité actuarielle de l'organisme fut atteinte seulement en 1918, par suite de l'amendement voté en 1916 pour obliger les mutuelles de son territoire à s'y conformer. Ontario. Statutes of Ontario, $6 \mathrm{Geo}$ V, (1916), An Act to amend the Ontario Insurance.Act, King's Printer, 1916, p. 171. 
importance diminua au fil des années avec l'augmentation de l'offre culturelle et la multiplication des associations. La participation active s'en trouva grandement restreinte dans les années 1880 et $1890^{53}$. Ainsi, le sociétaire développa un rapport de clientèle avec les sociétés de secours mutuel, surtout les nouveaux membres, qui n'avaient pas participé au projet social originel. L'intérêt pour la participation active s'en trouva fortement diminué chez la nouvelle génération. Le poids de ces sociétaires était important puisque les effectifs de l'association avaient doublé entre 1895 et 1897 à la suite de l'implantation de succursales dans la région d'Ottawa. Plusieurs d'entre eux n'avaient jamais connu la mutualité paroissiale, qui avait précédé la réforme de 1895. Pour les nouveaux sociétaires, le rapport de clientèle envers l'Union Saint-Joseph et la valeur du service financier supplantaient l'importance de la solidarité communautaire. D'ailleurs, ces membres avaient été admis après la transformation de la mission sociale, qui présentait l'association comme une initiative nationale visant à rallier le plus de Canadiens français dans ses rangs. Il faut également ajouter la contribution de la loi ontarienne de 1893, qui favorisa le développement de ce rapport de clientélisme en attribuant à la mutualité un statut relativement semblable à celui de l'assurance. Cette mutation des objectifs sociaux originels permit l'éclosion d'une nouvelle conception dominante de la mutualité, axée sur l'offre de services à la population canadienne-française par une certaine élite.

En plus des raisons financières, la nature du projet social que proposèrent les dirigeants contribua à l'acceptation des réformes. Ils jetèrent, en 1895, les bases d'une grande société de secours mutuel qui défendraient les valeurs canadiennes-françaises. Dans le premier numéro du journal officiel envoyé aux membres, les rédacteurs décrivirent ainsi le projet, tel qu'il existait lors de la réforme de $1895^{54}$ :

Notre devoir est tout tracé : travaillons pour l'Union Saint-Joseph ; qu'elle devienne forte et puissante et qu'elle compte parmi ses

53 Yvan Lamonde, Histoire sociale des idées au Québec, t. I, 1760-1896, Saint-Laurent, Fides, 2000, p. 478-480.

54 Les articles ne sont pas signés, mais proviennent du conseil fédéral, à Ottawa. 
membres le plus grand nombre de nos compatriotes d'Ontario ; qu'elle continue son travail jusqu'au jour où elle pourra prendre place parmi les sociétés importantes, et qui sait, si un jour ne vient pas, où toutes les sociétés Saint Joseph du Canada se donnant la main, se fusionneront, sous le titre de "Union Saint-Joseph du Canada " et continueront à secourir leurs membres et leur donner une assurance aussi considérable que n'importe quelle société étrangère ou compagnie d'assurance [sic], et à un taux aussi peu élevés5.

Concrètement, les sources concernant les transformations administratives de 1895 et de 1898 ne permettent pas de statuer clairement sur la force du facteur identitaire national dans l'acceptation des réformes. Toutefois, l'élite exprima clairement ses ambitions nationales en 1895. Durant cette période où les autorités provinciales tentaient d'angliciser l'éducation et menaçaient les bases de la communauté canadienne-française en Ontario, ces institutions nationales devenaient des instruments nécessaires à la survivance du groupe ${ }^{56}$. La situation minoritaire des Canadiens français de l'Ontario et les combats pour la survivance donnèrent des munitions pour convaincre les sociétaires de la nécessité d'adopter les réformes, au nom d'un idéal national. Ainsi, lorsqu'une petite élite proposa de transformer l'Union SaintJoseph en société provinciale afin de rassembler les Canadiens français de la province en une puissante organisation, les sociétaires acceptèrent. Le développement de succursales dans les communautés canadiennesfrançaises de la province dépourvues d'associations de prévoyance symbolisait plus qu'une réforme vers une forme de prévoyance plus stable ; le développement d'une structure nationale incarnait le désir de lutter pour renforcer les institutions canadiennes-françaises en Ontario.

55 L'Union Saint-Joseph d'Ottawa, Bulletin officiel, 15 juin 1895, p. 6.

56 Robert Choquette, La foi gardienne de la langue en Ontario, 1900-1950, Montréal, Les Éditions Bellarmin, 1987, p. 107-131. 


\section{La reconceptualisation du projet social de l'Union Saint-Joseph}

Lors de l'élaboration de la constitution de 1895, l'élite prit soin d'en retirer les clauses qui garantissaient son apolitisme. Concrètement, l'interdiction d'imposer des amendes depuis 1892 avait annihilé l'efficacité du processus disciplinaire contre les sociétaires qui aborderaient des sujets religieux et politiques. De plus, ces clauses étaient incompatibles avec la nouvelle direction que l'élite désirait donner à l'Union Saint-Joseph. Le retrait de ces restrictions permit l'adoption d'un nouveau discours basé sur le catholicisme social, à l'image des autres sociétés fraternelles catholiques. Ces associations bénéficiaient grandement de la publicité de leurs activités par les membres du clergé et les groupes ultramontains. D'ailleurs, à partir des années 1890, l'Église ne se contentait plus de simplement superviser la moralité des associations. L'exode des Canadiens français vers les États-Unis poussa le clergé à s'intéresser davantage à la condition économique de ses fidèles, en cherchant à offrir davantage que la charité pour encadrer la classe populaire ${ }^{57}$. La mutualité faisait partie de cette solution. $\mathrm{M}^{\mathrm{gr}}$ Fabre aurait d'ailleurs incité un groupe d'hommes d'affaires de Montréal à fonder l'Alliance nationale en 1892, une autre société fraternelle opérant un réseau de succursales ${ }^{58}$. George Emery et J. C. Herbert Emery, dans leur recherche sur l'Independent Order of Odd Fellows, constatèrent d'ailleurs une baisse de la participation des catholiques romains dans cette association neutre à partir des années 1880. Ils attribuent cette diminution au nouveau message social de l'Église, alors que le pape interdisait aux catholiques, en 1894, d'adhérer à certaines associations neutres, dont

57 William Ryan, The Clergy and Economic Growth in Quebec (1896-1914), Québec, Les Presses de l'Université Laval, 1966, p. 198.

58 Annie Desaulniers, Le secours mutuel en transformation : l'Alliance nationale (18921948), mémoire de maîtrise, Trois-Rivières, Université du Québec à Trois-Rivières, Département d'études québécoises, 2002, p. 34-35. 
les Odd Fellows, les Knights of Pythias ou encore les Sons of Temperance ${ }^{59}$.

Il n'existe aucune preuve de l'ingérence du clergé lors des réformes administratives de 1895 et de 1898 pour inciter l'Union Saint-Joseph à retirer de sa constitution les clauses qui interdisaient d'aborder des sujets religieux. Cependant, le chapelain démissionna en signe de protestation afin de montrer son désarroi devant l'incapacité de l'organisation à refondre sa constitution pour résoudre la crise en 1894. Les sociétaires s'empressèrent de voter une mesure, adoptée à l'unanimité, pour invalider sa démission et, ainsi, éviter de déplaire aux représentants de l'Église ${ }^{60}$. Il s'agit là des limites de l'information disponible concernant l'ingérence cléricale durant la période de réforme. Il est toutefois évident que si l'association voulait se transformer en une société provinciale ou nationale, elle devait adopter un discours inspiré du catholicisme social. L'absence de détails sur la pensée des réformateurs mutualistes d'Ottawa limite aussi les interprétations concernant les objectifs premiers, à savoir s'ils agissaient par conviction religieuse ou simplement pour assurer de nouvelles bases à l'Union Saint-Joseph en se conformant aux idées dominantes du moment, et, finalement, sauver leur certificat d'assurance. Dans les deux cas, cette élite n'avait d'autre choix que de se plier à la vision cléricale de la mutualité puisque le clergé encourageait ses fidèles à se joindre aux associations ouvertement catholiques qui promouvaient la doctrine sociale de l'Église. D'ailleurs, la Société l'Assomption, la mutuelle des Acadiens fondée au début du Xxe siècle, connut quelques difficultés à concurrencer les associations catholiques étrangères lors de son établissement à cause du manque d'appui du clergé. Euclide Daigle mentionne dans son historique sur cette société fraternelle que la reconnaissance officielle de la part de la province ecclésiastique

59 George Emery et J. C. Herbert Emery, A Young Man's Benefit : The Independent. Order of Odd Fellows and Sickness Insurance in the United States and Canada, 1860-1929, Montréal, McGill-Queen's University Press, 1999, p. 26-31.

60 Assemblée générale, Minute book, 1 novembre 1892 au 6 décembre 1897 , 6 novembre 1894, CRCCF, C20, vol. 12. 
d'Halifax vint seulement lorsque les succursales établies avant l'approbation de l'Église "firent acte de soumission ${ }^{61}$ ". Daigle demeure flou quant à la nature de cette soumission, mais il s'agissait probablement de la visite d'un aumônier et de l'adoption d'une ligne de conduite entièrement conforme aux valeurs catholiques. Ainsi, les dirigeants des mutuelles devaient emboîter le pas ou perdre les bonnes grâces du clergé au profit des sociétés de secours mutuel concurrentes. À Ottawa, l'arrivée des associations étrangères catholiques telles que les Forestiers catholiques et la Catholic Mutual Benefit Association contribua à accélérer ce rapprochement entre le discours de l'Union Saint-Joseph et celui de l'Église ${ }^{62}$. L'adoption d'une ligne de conduite inspirée de la doctrine sociale de l'Église pour y asseoir les valeurs mutualistes devint une manière de se greffer à un réseau institutionnel et profiter de la publicité offerte par le clergé. À l'inverse, la neutralité était impensable puisqu'elle aurait amené une condamnation de l'Église, ce qui aurait été inconcevable spirituellement et socialement, compte tenu de la participation de plusieurs dirigeants de l'Union Saint-Joseph aux activités paroissiales.

Le nouveau caractère religieux de l'Union Saint-Joseph poussa l'organisation à intensifier sa lutte contre les associations jugées dangereuses ${ }^{63}$. Le journal, Le Prévoyant, servit dans ce but, par la publication d'articles pour condamner les sociétés secrètes et non conformes aux idéaux de la doctrine sociale de l'Église. La politique développée par l'organisation consistait à formuler une catégorisation d'associations mutualistes pour les Canadiens français. Elle les divisa en deux principaux groupes : celles qui desservaient la nation et l'Église, et les étrangères ou non confessionnelles. Les textes

61 Euclide Daigle, Petite histoire d'une grande idée, Moncton, Imprimerie Acadienne, 1978 , p. 39-44.

62 Le Bulletin officiel, c'est-à-dire le journal de l'Union Saint-Joseph, adopte un discours dans ses articles qui se rapproche de la doctrine sociale de l'Église. Il s'agit d'une transition importante pour une association apolitique et dont les règlements interdisaient d'aborder des sujets religieux avant 1895 .

${ }^{63}$ Ibid., p. 7. 
condamnaient les Canadiens français qui adhéraient à ces dernières ${ }^{64}$. Ainsi, l'Union Saint-Joseph garantissait une supériorité morale en répondant à la fois à un besoin spirituel et économique. L'adoption de cette mission inspirée du catholicisme social facilita considérablement le recrutement. Le clergé, souvent encouragé par $\mathrm{M}^{\mathrm{gr}}$ Duhamel, accueillait à bras ouverts les administrateurs de l'Union Saint-Joseph lors de la fondation de nouvelles succursales, en offrant de parler après la messe ou en publicisant les assemblées ${ }^{65}$. Le nombre de sociétaires augmenta considérablement grâce à cette stratégie, passant de 1580 en 1898 à 17944 en 1906, alors que les succursales se chiffraient à 337 la même année ${ }^{66}$.

Cette croissance de l'Union Saint-Joseph et le prolongement de ses activités dans les communautés francophones les plus importantes du Canada et des États-Unis eurent pour effet de renforcer la conscience nationaliste des dirigeants. Le sentiment de participer à une œuvre visant à unir les Canadiens français existait dès 1895, mais les crises scolaires en Ontario poussèrent la rhétorique à un niveau supérieur. C'est à la fin des années 1900, plus précisément en 1908, que la transition finale vers une organisation nationaliste atteint son apogée. Un nouveau président, G. W. Séguin, prit la tête de l'Union Saint-Joseph. Ce dernier œuvrait au sein de l'association depuis plus de vingt-cinq ans et avait occupé plusieurs postes administratifs dans différents comités. Grand nationaliste, il fut connu plus tard pour sa participation à la fondation de l'Association canadienne-française

${ }^{64}$ L'Union Saint-Joseph d'Ottawa, Bulletin officiel, 15 mai 1896, p. 63.

${ }^{65}$ Il y a plusieurs mentions de l'accueil chaleureux qu' ont reçu les membres de l'Union Saint-Joseph. Par exemple, ils sont accueillis à Rockland par des paroissiens, le curé et la Société Saint-Jean-Baptiste. La situation est semblable à Embrun, à Clarence Creek et autres endroits visités. Voir, par exemple, Minutes book, bureau de direction, 7 février 1895 au 11 novembre 1896, 11 mars 1895, CRCCF, C20, vol. 18.

${ }^{66}$ Ontario. Ontario Sessional Papers 1900, The Report of the Inspector of Insurance (1898), vol. XXXII, part III, n ${ }^{\circ} 10$, p. C204 ; Ontario. Ontario Sessional Papers 1907, The Report of the Inspector of Insurance (1906), vol. XXXIX, part III, $\mathrm{n}^{\circ} 10, \mathrm{p} . \mathrm{C} 221$. 
d'éducation d'Ontario (ACFEO). Dans son premier discours, il déclara vouloir donner un rôle national à l'Union Saint-Joseph dans la sauvegarde de la "race " canadienne-française. Séguin insistait, cependant, sur un aspect en particulier :

L'action sociale vise surtout les générations futures. C'est pourquoi son premier champ d'opérations est ou doit être l'école, l'école catholique et, particulièrement dans la province d'Ontario, l'école française, ou comme nous sommes convenus de l'appeler pour faire taire certains préjugés puissants, l'école bilingue. La sphère dans laquelle la St-Joseph doit mettre en œuvre ses ressources et son influence est donc toute délimitée. C'est sur l'école que doit s'exercer sa vigilance, s'étendre sa sollicitude ${ }^{67}$.

Il serait faux d'affirmer que l'augmentation du militantisme de l'organisation résultait seulement de l'élection de Séguin. Les réformateurs, qui étaient demeurés en place après la transformation administrative et actuarielle, avaient quitté graduellement la direction de l'Union Saint-Joseph, ouvrant la porte à une nouvelle administration, plus dévouée à la cause nationale. Si Séguin était très engagé dans la question scolaire, il n'était pas le seul. Le sénateur Napoléon-Antoine Belcourt, premier président de l'ACFEO, avait œuvré comme avocat de l'Union Saint-Joseph durant les années 1890 et conservait beaucoup de liens avec celle-ci. C'est d'ailleurs lui qui, en 1905 - alors qu'il était député -, avait présenté au Parlement la demande pour changer le nom de l'Union Saint-Joseph d'Ottawa en Union Saint-Joseph du Canada $^{68}$. De plus, la multiplication des succursales avait permis à l'association d'accroître cette conscience nationale, par l'ajout d'effectifs répartis dans l'ensemble des régions francophones du pays.

67 L'Union Saint-Joseph du Canada, Le Prévoyant, vol. XIV, n 1, novembre 1908, p. 2.

68 Débats de la Chambre des communes : compte rendu officiel, Ottawa, Imprimeur de la Reine, 1905, p. 2598 ; Canada. Statutes of Canada, Ed. 7 (1905), An Act respecting l'Union Saint-Joseph de la cité d'Ottawa, and to change its name to l'Union SaintJoseph du Canada, Ottawa, Samuel Edward Dawson, King's Printer, p. 517. 
Le mois suivant la déclaration de Séguin, Le Prévoyant, le journal de l'Union Saint-Joseph, modifia son format et commença à publier des éditoriaux à saveur plus patriotique. Le journal propagea une idéologie d'entraide axée sur le sentiment d'identité canadiennefrançaise et intensifia sa lutte non seulement contre les associations non catholiques, mais aussi contre les associations anglophones catholiques. En 1910, les objectifs étaient clairement définis, et son militantisme s'alimentait du besoin de renforcer les bases nationales pour défendre les droits des Canadiens français. Les crises scolaires et, surtout, le Règlement 17 en Ontario, qui limitait l'enseignement du français dans les écoles de la province, touchaient une partie de l'élite dirigeante de l'Union Saint-Joseph ainsi que la moitié de ses sociétaires $^{69}$. La sauvegarde de la nation allait dépendre de la capacité des Canadiens français à s'unir. Les dirigeants de l'Union Saint-Joseph affirmaient qu'il fallait également éviter la prise de contrôle des capitaux canadiens-français par les associations anglo-saxonnes, d'où l'importance de militer contre l'enrôlement des Canadiens français dans les associations étrangères. Les réformateurs mutualistes espéraient ainsi participer à la cause tout en recrutant plus de sociétaires. Il ne s'agissait pas seulement d'animer un sentiment identitaire dans le but d'attirer des aspirants, mais aussi de s'insérer dans un projet nationaliste dans l'espoir d'y établir des bases solides pour l'Union Saint-Joseph, comme ce fut le cas avec la doctrine sociale de l'Église en 1895.

Certains ajustements furent nécessaires pour adapter l'association à ces nouveaux objectifs. Un travail de redéfinition de la raison d'être de l'Union Saint-Joseph s'effectua, allant même jusqu'à une réinterprétation du passé et des débuts de l'organisation pour conso-

69 En 1910, sur les trente-six administrateurs les plus haut placés, dix-huit habitaient l'Ontario, ce qui explique pourquoi cette question représentait un aspect important de "l'œuvre patriotique ". L'Union Saint-Joseph du Canada, Le Prévoyant, vol. XV, $\mathrm{n}^{\circ} 18$, juin 1910 , p. 56. 
lider le sentiment patriotique ${ }^{70}$. En 1908, on pouvait lire dans Le Prévoyant: "Notre société est née aussi d'une idée religieuse et patriotique. Ne lit-on pas à l'entête [sic] de ses publications, de ses circulaires, de ses annonces qu'elle est exclusivement catholique et canadienne-française ${ }^{71}$ ? " Bien sûr, l'Union Saint-Joseph était née d'un mouvement canadien-français, mais elle avait pour objectif, à ses débuts, de secourir la classe populaire canadienne-française en offrant une alternative à la charité et non de promouvoir les idées patriotiques comme c'était le cas en 1910. Cette révision de sa propre histoire cherchait à légitimer les activités de l'organisation, en lui prétendant une origine nationale dès sa fondation. Cette propagande nationaliste représentait, cependant, les limites de son œuvre hors de la sphère économique. L'ensemble de ses activités se concentrait autour de la mutualité. La seule initiative hors de ce domaine, quoiqu' importante, fut l'utilisation de son réseau de succursales pour l'envoi de lettres circulaires invitant les Canadiens français de l'Ontario au congrès qui eut lieu dans la salle de l'Union en 1910 et qui marqua la fondation de l'ACFEO ${ }^{72}$. Cependant, la collaboration de l'Union Saint-Joseph avec cette dernière fut de courte durée en raison de querelles entre les présidents respectifs des deux associations. En fait, c'est beaucoup plus la cause nationale qui servit le prestige de l'Union Saint-Joseph que l'inverse. Le rapport des administrateurs de 1911 témoigne de cette situation :

Nos nombreux groupements existant dans les diverses sections de la province ont permis l'inauguration de cette association patriotique en peu de temps. Si les Canadiens français d'Ontario ont beaucoup

70 On peut le constater en lisant les livres de Charles Leclerc sur l'histoire de l'Union Saint-Joseph ou ses articles dans Le Prévoyant. L'accent est mis sur la question nationale canadienne-française, même si ce n'était pas le cas durant les deux premières décennies de son histoire. Leclerc, L'Union Saint-Joseph du Canada, 89 p. ; L'Union Saint-Joseph du Canada, Le Prévoyant, vol. XIV, n 1 , novembre 1908 , p. 1.

71 Ibid.

72 Gaétan Gervais, Des gens de résolution: le passage du "Canada français " à l' "Ontario français ", Sudbury, Institut franco-ontarien, 2003, p. 60. 
bénéficié de la création de cette association, il faut, en toute justice, dire que l'Union St-Joseph du Canada, par l'entremise de cette coopération, a pu recruter un plus grand nombre d'adhérents et a ajouté considérablement à son prestige dans la province ${ }^{73}$.

En somme, l'Union Saint-Joseph avait peu d'outils pour participer activement aux luttes sociales. La fin de la présence obligatoire aux assemblées, en 1895, avait diminué son véritable pouvoir d'action, lequel reposait sur la force de son unité. Ses sociétaires ne participaient presque plus aux activités depuis l'introduction d'une relation de clientèle, à l'exception de l'élite dirigeante et de quelques intéressés. Devant cette faible capacité d'action, la propagande nationaliste engendra peu d'initiatives concrètes à l'extérieur du domaine mutualiste. L'association se limitait à ce qu'elle faisait le mieux, soit offrir une assurance à bon prix ainsi qu'une solution de rechange aux associations anglo-saxonnes ou étrangères.

\section{Conclusion}

Ce que les premiers mutualistes craignaient lorsqu'ils établirent le fondement de la première génération de sociétés de secours mutuel, c'est-à-dire la perte de contrôle des sociétaires au profit d'une élite instruite, se produisit. La constitution et les règlements n'ont pas réussi à protéger complètement l'association, mais ils avaient maintenu en place des mécanismes devenus désuets durant les années 1890 par rapport aux idées mutualistes dominantes et à la volonté d'une partie des membres de ne plus participer activement au fonctionnement des sociétés de secours mutuel. De plus, la structure avait prolongé l'existence d'une gestion financière qui menait à l'insolvabilité, de sorte que l'association devait revoir l'ensemble de sa façon d'administrer la mutualité. Cette crise, qui émergea pendant les années 1890, ouvrit la porte à une refonte du modèle mutualiste originel, inspiré de l'Union Saint-Joseph de Montréal, pour l'adapter à la réalité des

73 Septième session du Conseil fédéral, août 1911, Rapports des officiers, p. 56, CRCCF, C20/B3/2. 
Canadiens français d'Ottawa. L'Union Saint-Joseph d'Ottawa, à l'image des autres sociétés de secours mutuel de première génération, se nourrissait du contexte dans lequel évoluaient ses membres pour forger sa mission sociale. Toutefois, cette tentative de réforme échoua dans d'autres localités, forçant plusieurs mutuelles à déclarer faillite ou à fusionner avec des associations fraternelles.

Les conditions qui permirent la réussite de cette mutation à Ottawa provenaient, en grande partie, du contexte dans lequel évoluaient ses membres et du climat mutualiste qui existait dans la ville à cette période. Le rapport plus commercial qui se développa entre les sociétaires et l'association suivant sa croissance rapide durant les années 1880 et 1890, l'interdiction par le gouvernement ontarien d'imposer des amendes, ainsi que la multiplication des succursales entre 1895 et 1898, rendaient pratiquement impossible la continuité des anciens objectifs de solidarité et d'entraide. Ainsi, comme ce fut le cas lorsque les travailleurs forgèrent la première structure administrative de l'Union Saint-Joseph de Montréal, l'élite, appelée à trouver une solution, puisa dans les forces sociales dominantes du moment pour établir une doctrine mutualiste permettant de rallier le plus de gens possible et prolonger son existence. Le rôle que l'Église jouait depuis cinquante ans pour maintenir les institutions canadiennesfrançaises à Ottawa et, plus tard, pour le droit à l'enseignement en français ne pouvait qu'inspirer les réformistes et les dirigeants. Pour les sociétaires, la nécessité de se rallier au réseau institutionnel catholique pour défendre la culture canadienne-française justifiait la transition, tout comme le développement de succursales dans le but de réunir le plus de Canadiens français possible sous une même bannière. La prise de contrôle par quelques dirigeants à la suite des réformes fut semblable dans les autres milieux d'immigration, comme le constatent John Bodnar et Yves Roby dans le cadre des expériences migratoires aux États-Unis. Dans la plupart des cas, c'est à cette élite que revenait le rôle d'assurer les assises culturelles de ces nationalités nouvellement arrivées dans une région. 
Pendant la soixantaine d'années qui suivit sa transformation de 1895, l'Union Saint-Joseph d'Ottawa continua de s'appuyer sur des bases nationalistes et religieuses pour alimenter son projet social. L'élite put diriger l'association sans trop d'opposition puisque peu de sociétaires participaient au processus décisionnel. Elle choisit ainsi les combats sociaux qu'elle désirait mener, et put modifier le message selon les besoins économiques et nationalistes du moment, toujours dans les limites des compétences mutualistes. Autrement dit, à l'exception de sa mission d'éducation et de propagande, remplie par la publication de son journal, sa tâche dans le réseau institutionnel canadien-français se résumait à offrir un service aux Canadiens français pour éviter leur adhésion aux associations anglo-saxonnes et enrichir les compagnies d'assurances. Cette transformation assura toutefois la pérennité de l'Union Saint-Joseph, dont la force et les bases nationalistes lui permirent de prolonger son existence pendant tout le $\mathrm{xx}^{\mathrm{e}}$ siècle. 